\title{
Die eidgenössische Prüfung ist keine Lehrveranstaltung
}

\section{Sissel Guttormsen ${ }^{\mathrm{a}}$, André Perruchoud ${ }^{\mathrm{b}}$}

a Prof. Dr. phil., Direktorin Institut für Medizinische Lehre, Universität Bern; ${ }^{b}$ Prof. Dr. med., Präsident der eidgenössischen Prüfungskommission für Humanmedizin

Wir danken Herrn Prof. Probst und Herrn Huber für ihre ausführlichen Überlegungen zur eidgenössischen Prüfung (EP). Viele ihrer Einsichten teilen wir. Wir haben bereits mit unseren Beiträgen in der SÄZ relevante Aspekte beleuchtet [1, 2].

Grundsätzlich bezweifeln Herr Probst und Herr Huber den Mehrwert der Prüfung und wünschen sich stattdessen eine offene Prüfung mit echten Patienten und mehr Flexibilität, die gleichzeitig auch als eine Art Lehrveranstaltung dienen kann.

\section{Aktuell laufen Pilotprojekte mit Beteiligung von Kindern in standardisierten klinischen Prüfungen.}

Die neue EP findet nach Abschluss des Studiums und vor Eintritt in die Weiterbildung statt, wird von dem Medizinalberufegesetz (MedBG) gesteuert und von den Fakultäten durch ihre klinischen Experten inhaltlich vorbereitet. Die EP kommt zu spät, um als Lehrveranstaltung zu dienen. Sie ist eine letzte unabhängige Qualitätssicherungsmassnahme vor dem Eintritt in die klinische Arbeit unter Anleitung.

Mit dem neuen MedBG wurde die Ausbildung ganz in die Verantwortung der Fakultäten übergeben. Im neuen System finden an den Fakultäten verschiedene Prüfungen während des Studiums statt. Wir fragen uns, warum die Herren Probst und Huber gerade bei der EP so viel Wert auf die Weitergabe von Erfahrung und freie Prüfungen legen? Interaktionen mit Patienten, Üben und Testen verschiedener klinischer Fertigkeiten sollen während der Ausbildungsjahre praktiziert werden. Rückmeldungen von Studierenden zeigen mit grosser Konstanz, dass sie sich mehr Zeit, Anleitung und Feedback von erfahrenen klinisch tätigen Ärzten während der Ausbildung wünschen. Die EP wirkt auf das Lernverhalten und auf die gesetzten Schwerpunkte in der Ausbildung ein. Durch die transparente Bekanntmachung der Lernziele und Prüfungsthemen wissen die Studierenden und die Dozen- ten, welches Wissen und welche praktischen und kommunikativen Fertigkeiten für den Abschluss des Studiums erwartet werden und gezielt üben sollen. Die EP ist ein Spiegel der nationalen gesundheitspolitischen medizinischen Ausbildungsziele.

Die EP entwickelt sich ständig weiter und die Möglichkeiten, realitätsnahe Settings zur Verfügung zu stellen, ebenso. Aktuell laufen Pilotprojekte mit Beteiligung von Kindern in standardisierten klinischen Prüfungen; Hautkrankheiten können mit der Unterstützung von professionellen Maskenbildnern zum Verwechseln echt nachgebildet werden; Notfallszenarien können mit Monitoren, die auf Interventionen reagieren, nachgestellt werden; intelligente Stethoskope können klinische Herztöne abhängig von der Abhörstelle wiedergeben; hybride Kombinationen von Mensch und Modell bieten neue Möglichkeiten, klinischen Befunde darzustellen. Der Einsatz von Simulationen ermöglicht zudem, auch Aspekte zu prüfen, die mit realen Patienten in einer Prüfungssituation nicht möglich sind (z.B. Behandlung akuter Notfälle inkl. Wiederbelebungsmassnahmen).

\section{Intelligente Stethoskope können klinische} Herztöne abhängig von der Abhörstelle wiedergeben.

Die Prüfung steuert, was die Studierenden während der Ausbildung üben und aktiv lernen. Das sollte unseres Erachtens im Zentrum stehen und nicht die freie Prüfungssituation. So gesehen kann auch die Bedeutung der absoluten Realität relativiert werden. Für das Expertenfeedback und den direkten Bezug zur klinischen Realität bieten das Studium und die Weiterbildung nicht nur die besseren, sondern optimale Bedingungen.

\section{Referenzen}

1 Guttormsen S, Perruchoud A. Pionierarbeit für die Abschlussprüfung Medizin. Schweiz Ärztezeitung 2015;96(50-51):1846-8.

2 Guttormsen S, Perruchoud A. Die neue eidgenössische Prüfung hat einen Mehrwert. Schweiz Ärztezeitung 2016;97(4):155-6. 